\title{
Influence of the nature of fluoropolymer processing aids on the elimination of sharkskin defect in LLDPE extrusion
}

\author{
C. Dubrocq-Baritaud ${ }^{1}$, N. Devaux ${ }^{2}$, E. Darque-Ceretti ${ }^{1}$, B. Vergnes $^{1}$ \\ ${ }^{1}$ CEMEF, Ecole des Mines de Paris, UMR CNRS 7635 - BP 207, 06904 Sophia Antipolis, France \\ URL: www.cemef.cma.fr \\ e-mail: claire.dubrocq@ensmp.fr; \\ evelyne.darque-ceretti@ensmp.fr; \\ bruno.vergnes@ensmp.fr;
}

${ }^{2}$ CERDATO, Arkema - 27470 Serquigny, France

URL: www.arkema.fr

e-mail: nicolas.devaux@arkemagroup.com;

\begin{abstract}
Fluoropolymer-based processing aids (PPA) are commonly used to suppress sharkskin defects in polyolefin extrusion. PPA's also lead to a decrease in die pressure and a slight increase in flow rate by coating die walls and inducing wall slip. The aim of this work is to investigate the influence of different types of PPAs on the physical parameters in the die at various scales. We used a modular slit die fed by an extruder to measure die pressure and velocity profiles by Laser Doppler Velocimetry (LDV), and to characterize die surface coating by Scanning Electronic Microscopy (SEM).
\end{abstract}

Key words: Sharkskin defect, Polymer processing aids, Fluoropolymer, Extrusion.

\section{INTRODUCTION}

Surface defects (sharkskin) are the most limiting polymer flow instability since they appear at low production rates in extrusion processes [1, 2]. For twenty years, fluoropolymer-based processing aids (PPA) are commonly used to suppress sharkskin in polyolefin extrusion, but also to decrease die pressure [3]. In a previous study, we investigated the kinetics of action of a PPA by following die pressure, surface aspects of extrudates, wall slip velocity and deposits of fluoropolymer on the die surface [4]. In the present paper, we are interested in the influence of the PPA nature on the mechanisms of elimination of sharkskin defects.

\section{MATERIALS AND METHODS}

\subsection{Materials}

A commercially available linear-low density polyethylene (LLDPE Innovex ${ }^{\circledR}$, produced by Ineos) was selected for its wide range of shear rate where sharkskin defects appear.

The first two tested PPA's (FP-1 and FP-2) are pure thermoplastic copolymers of vinylidene and hexafluoropropene (KynarFlex ${ }^{\circledR}$, produced by Arkema).
The last one (FP-2+PEG) is a mechanical blend of FP-2 with polyethylene glycol (PEG) $\left(\right.$ KynarFlex $^{\circledR}$, Arkema). The physical characteristics of all resins are given in Table I.

Homogeneous masterbatches of $5 \%$ of the PPA's in LLDPE (called MM 5\%) were blended in a twinscrew extruder.

Table1. Physical characteristics of the resins

\begin{tabular}{lcccc}
\hline & LLDPE & FP-1 & FP-2 & (FP-2+PEG) \\
\hline $\begin{array}{l}\text { Melt temperature } \\
{ }^{\circ} \mathrm{C}\end{array}$ & 122 & 146 & 164 & $>60$ \\
$\begin{array}{l}\text { Density } \\
\mathrm{g} / \mathrm{cm}^{3}\end{array}$ & 0.920 & 1.800 & 1.790 & 1.500
\end{tabular}

\subsection{Experimental set-up for extrusion experiments}

Experiments were carried out on a laboratory singlescrew extruder Brabender $(L / D=25, D=19 \mathrm{~mm})$ in line with a transparent slit die unit. Figure 1 represents the die system where the die contraction is made up of two removable inserts [4]. The steel inserts are surfaced parallel to the flow direction. Roughness parameters are $R_{a}=0.5 \mu \mathrm{m}$ and $R_{z}=3.8$ $\mu \mathrm{m}$. Extrusion speed is fixed at $60 \mathrm{rpm}$ and die temperature at $180^{\circ} \mathrm{C}$. The corresponding experimental conditions are: a melt temperature of $186^{\circ} \mathrm{C}$, an apparent wall shear rate of $135 \mathrm{~s}^{-1}$ and an apparent wall shear stress of $0.2 \mathrm{MPa}$. 


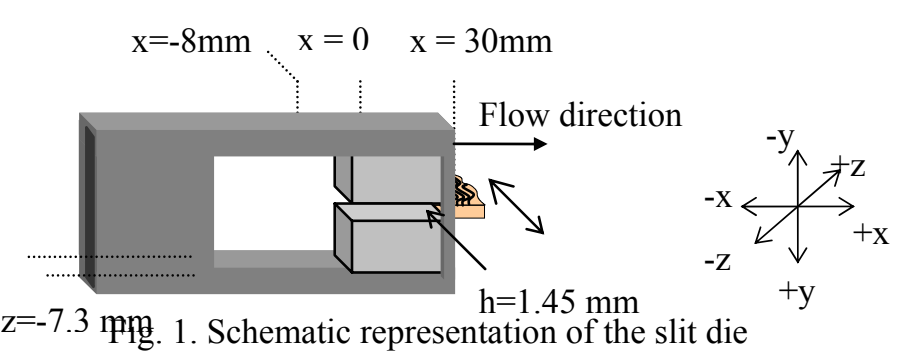

First, pellets of MM 5\% PPA and of pure LLDPE are tumble-blended to obtain a typical concentration of $250 \mathrm{ppm}$ PPA. Then, extrusions with PPA are performed after stabilization of the flow with pure LLDPE (PPA is introduced at time 0). The flow rate is evaluated from extrudates collected at the die exit during $36 \mathrm{~s}$ and weighted off-line. Samples are also collected and cooled in ambient air to quantify offline the sharkskin defects.

The total extrudate width where sharkskin defects are present is measured and normalized by the sample width. During certain extrusion experiments, Laser Doppler Velocimetry (LDV) technique is used to measure velocity profiles in the slit die. At the end of each experiment, the inserts are removed and quenched in water for die surface analyses by Scanning Electronic Microscopy (SEM).

\section{RESULTS AND DISCUSSION}

\subsection{Effect of PPA on macroscopic extrusion parameters}

The evolution of die pressure and sharkskin defects on extrudate surface is shown in figure 2. Figure 2(a) presents the decrease in die pressure for the three PPA's. Whatever the fluoropolymer, the pressure follows a sigmoidal curve with a constant induction time $\left(t_{0}=135 \mathrm{~s} \pm 15 \mathrm{~s}\right)$. This time is higher than the mean residence time in the extruder $\left(t_{r}=\right.$ $105 \mathrm{~s})$. Die pressure decreases more rapidly for FP2+PEG, followed by FP-2 and by FP-1. However, FP-1 and FP-2 have the same final normalized pressure $\left(P_{f} / P_{0}\right)$ whereas (FP-2+PEG) presents a lower value. Besides, the new equilibrium seems to be reached faster for FP-2 than for the other PPA's.

Concerning the elimination of the sharkskin defects, the curves are ranked in the same order, with a defect that successively disappears for FP-2+PEG, FP-2 and FP-1. One can notice that only FP-1 shows a real sigmoid-type curve for sharkskin defect elimination.

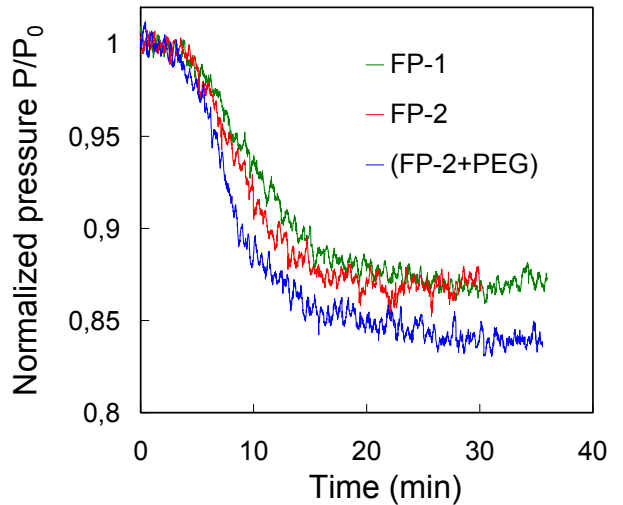

(a)

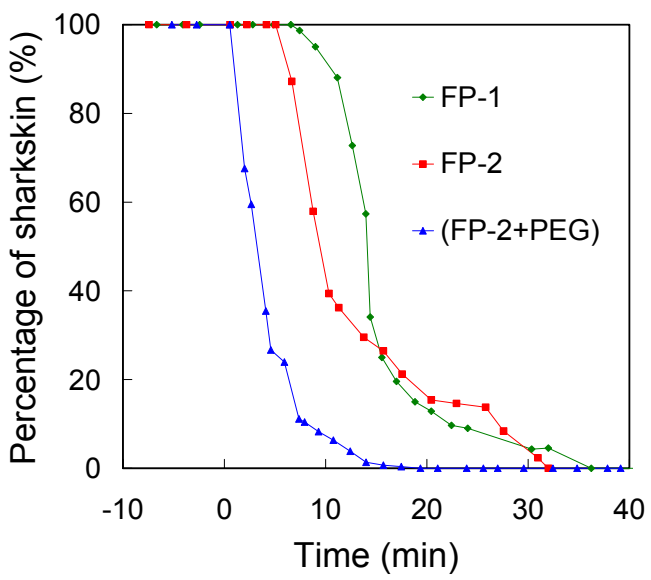

(b)

Fig. 2. Effect of 250 ppm PPA on the evolution of macroscopic parameters of extrusion. (a) Die pressure normalized by its initial value. (b) Percentage of sharkskin on the extrudate surface.

For the other PPA's, the disappearance of sharkskin is so rapid that it is not experimentally possible to collect enough samples to accurately quantify the early beginning of the sharkskin elimination.

Table2. Characteristics of the new equilibrium

\begin{tabular}{lcccc}
\hline & & FP-1 & FP-2 & (FP-2+PEG) \\
\hline$P_{f} / P_{0}$ & $\%$ & $87 \pm 0.5$ & $87 \pm 0.5$ & $83 \pm 0.5$ \\
$t_{\text {no sharkskin }}$ & min & $36 \pm 1$ & $32 \pm 1$ & $17 \pm 1$ \\
$Q_{f} / Q_{0}$ & $\%$ & $1.9 \pm 1.5$ & $2.5 \pm 1.5$ & $2.7 \pm 1.5$ \\
\hline
\end{tabular}

Table 2 represents the principal characteristics of the macroscopic parameters in the new steady state reached with the three PPA's. In particular, it turns out that, during die pressure decrease and sharkskin elimination, the flow rate slightly increases for all fluoropolymers, somewhat more for FP-2+PEG and a little for FP-1.

\subsection{Morphology of coating}

When the new equilibrium is reached, inserts with PPA deposits are collected and the die surface is observed by SEM. Figure 3 displays the deposits of fluoropolymer at the centre of the die surface for the 
three PPA's. SEM images of the tilted die surface, shown on figure 3(a), allow the analyze of the shape and the thickness of PPA deposits, whereas BSE (Back Scattering Electron) images on figure 3(b) display the spatial organization and the density of the deposits. In addition, EDX (Energy Dissipative $\mathrm{X}$-ray) analyses confirm that the deposits observed in dark color with the BSE detector are composed of fluoropolymers.

FP-1

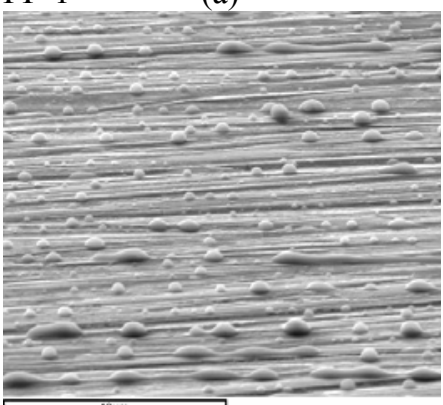

FP-2

(a)

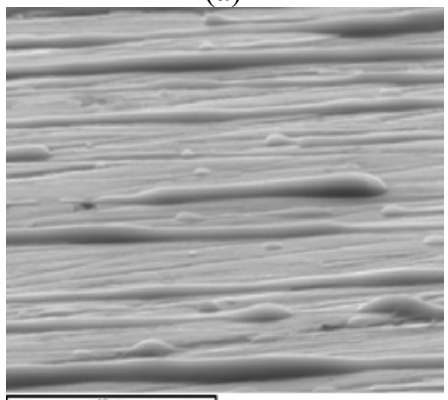

(FP-2+PEG)

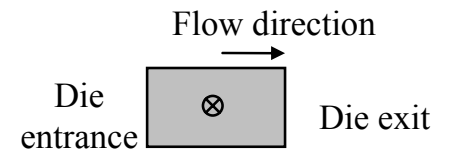

Fig. 3. PPA coating at the centre of the die surface observed by SEM, $20 \mathrm{keV}$. (a) SE detector, surface tilted $70^{\circ}$. (b) BSE detector.

For all PPA resins, the coating is spatially discontinous and its morphology preferentially oriented parallel to the flow direction. However, the three fluoropolymers show very different structures at the die walls. FP-1 coats the surface as lines of drops and short lines of fluoropolymer 70-100 $\mu \mathrm{m}$ long. The FP-2 coating is mainly composed of interconnected lines 3-5 $\mu \mathrm{m}$ wide and the FP-2+PEG coating is an interconnected network of $10 \mu \mathrm{m}$ wide strips. Tilted SEM images reveal that heterogeneous deposits are $1.9 \pm 0.2 \mu \mathrm{m}$ thick for FP-1 and $3.2 \pm$ $0.4 \mu \mathrm{m}$ thick for FP-2. It was not possible to observe the deposit thickness for FP-2+PEG since it is inferior or equal to the die surface roughness. Complementary analyses demonstrate that FP$2+$ PEG coating is nearly $1 \mu \mathrm{m}$ thick and thus the thinnest.

Besides, the observation of the BSE images reveals that there is a larger surface of deposits with FP2+PEG than with FP-2 and FP-1. Furthermore, as seen in a previous study on FP-2 [4], it is possible to verify that the surface density of PPA coating increases:

- from the die entrance to the die exit

- from the lateral edge to the middle of die width.

These last two points may explain why the final pressure is lower for the PPA with PEG.

\subsection{Flow conditions at the die walls}

\section{3.a Evolution of wall slip velocity}

In order to follow the effect of coating on the flow conditions at the die walls, LDV experiments were carried out inside the slit. Measured velocity profiles $V_{x}(y, t)$ were fitted with a power law (1) to obtain the velocity at the wall $V_{x}^{s}$ at various times.

$V_{x}(y, t)=V_{x}^{s}(t)+\left(V_{x}^{\max }(t)-V_{x}^{s}(t)\right)\left[1-\left(\frac{2 y}{h}\right)^{1+1 / n}\right]$

$V_{x}^{\max }$ is the maximal velocity, measured in the centre of the slit. The power law index $n$ is deduced from the viscosity curve of LLDPE at $135 \mathrm{~s}^{-1}$ and $180^{\circ} \mathrm{C}$. It is equal to 0.42 .

The evolution of pressure and velocity at the wall at different positions $x$ are represented in figure 4 for all PPA's. Whatever the PPA, the curves show that wall slip suddenly appears, at the same time as the pressure drops. Besides, a slight wall slip first appears on the first part of the die surface $(x=4 \mathrm{~mm}$ and $x=15 \mathrm{~mm})$, then on its second part $(x=26 \mathrm{~mm})$. We can thus deduce from the kinetic evolution of the velocity at the wall that the phenomenon at the origin of the migration of fluoropolymer is the same for all PPA's. 


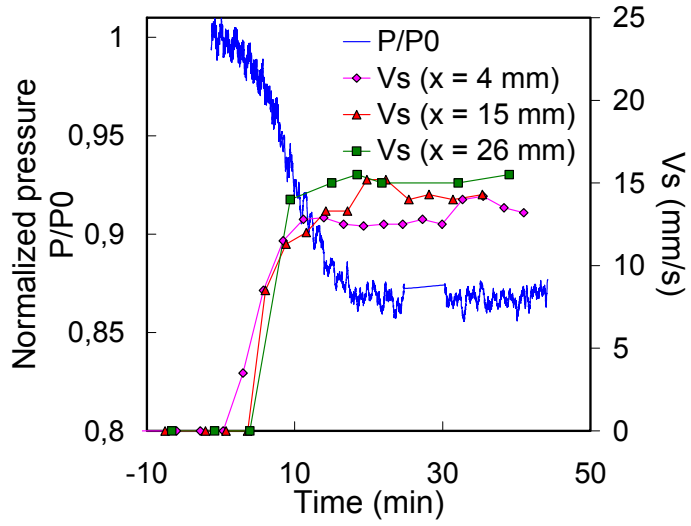

(a)

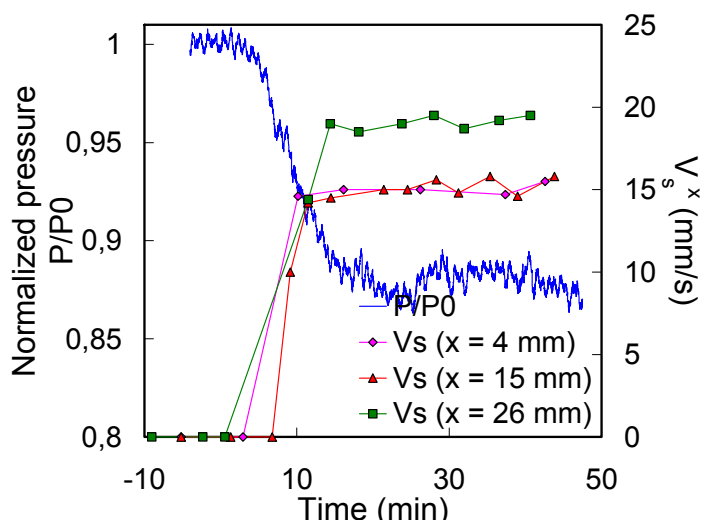

(b)

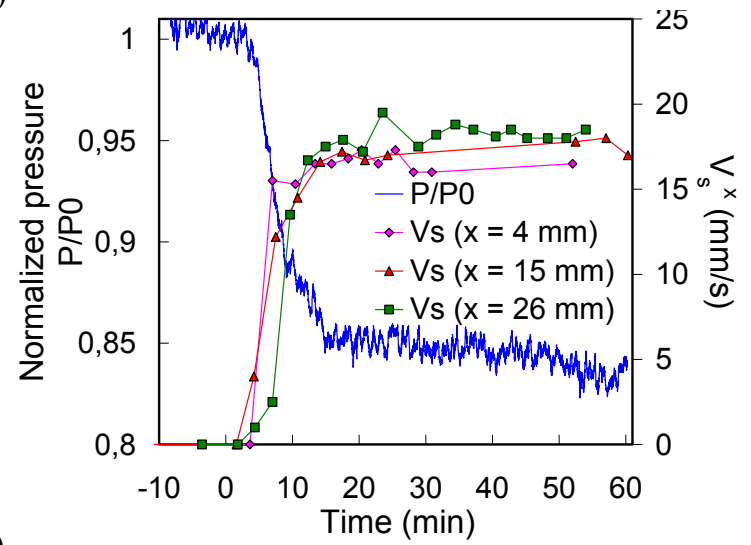

(c)

Fig. 4. Evolution of the velocity at the wall at different positions along the die (at $z=0$ ) for (a) FP-1, (b) FP-2 and (c) (FP-2+PEG).

\section{3.b Velocity at the wall in the new steady state}

From the last graphs, mean values of wall slip in the last twenty minutes can be deduced, at each position. For all PPA's, figure 5 reveals that:

- along the die, the wall slip increases from the entrance to the exit;

- across the die, the wall slip is lower at the lateral edge than in the middle.

Consequently, the results demonstrate that the evolution of wall slip inside the die is similar to the evolution of the coating surface density.

However, wall slip values are quite different for the three PPA's. Wall slip is globally higher for FP$2+\mathrm{PEG}$, which could explain its highest pressure drop. Wall slip for FP-1 follows the same behaviour as for FP-2+PEG, but with lower values. On the contrary, wall slip for FP-2 varies markedly: it is much lower at $(x=15 \mathrm{~mm}, z=4.8 \mathrm{~mm})$ than FP-1 value, higher at $(x=26 \mathrm{~mm}, z=0)$ than FP-2+PEG value and intermediate at $(x=4$ and $15 \mathrm{~mm}, z=0)$. This means that different wall conditions can lead to the same final die pressure in the new steady state reached with FP-1 and FP-2.

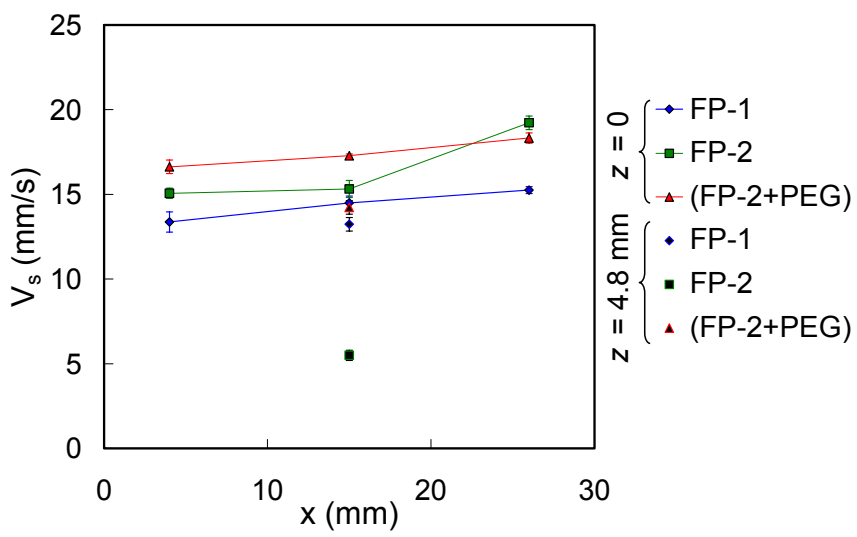

Fig. 5. Comparison of the stabilised wall slip velocity for the different PPA's.

\section{CONCLUSIONS}

The objective of the present work was to compare the effects of three PPA's on extrusion physical parameters, from the microscopic to the macroscopic scale. A quantification of PPA coatings was undertaken to confirm the direct link between the local density of coating and the local wall slip. It was found that the efficiency of PPA can be analysed, thus clearly putting in evidence the specific role played by the PEG.

\section{ACKNOWLEDGEMENTS}

The authors would like to thank Thierry Collin, for his help in optimizing and purging the slit die.

\section{REFERENCES}

1. Hatzikiriakos, S.G., Migler, K B., Polymer Processing Instabilities: Understanding and Control, Marcel Dekker, New York (2004).

2. N. El Kissi, J.M. Piau, 'Adhesion of linear low-density polyethylene for flow regimes with sharkskin', J. Rheol., 38 (1994) 1447-146

3. 3. S. Nam, 'Mechanism of fluoroelastomer processing aid in extrusion of LLDPE', Intern. Polym. Proc., 1 (1987) 98-101.

4. C. Dubrocq-Baritaud, N. Devaux, E. Darque-Ceretti and B. Vergnes, 'Mécanismes d'action des «Polymer Processing Aids» dans l'élimination du défaut de peau de requin en extrusion', Rhéologie, 12 (2007) 19-26. 